\title{
The concept of measurement of calorific value of gaseous fuels
}

\author{
Jaroslaw Markowski ${ }^{1, *}$, Pawel Imilkowski ${ }^{1}$, Marcin Nowacki ${ }^{1}$, Damian Olejniczak ${ }^{1}$, \\ Jacek Madry ${ }^{1}$, Krzysztof Netter ${ }^{1}$, Krzysztof Jesionek ${ }^{2}$, and Grzegorz Wieczorkiewicz ${ }^{3}$ \\ ${ }^{1}$ Poznan University of Technology, Faculty of Mechanical Engineering, Poland \\ ${ }^{2}$ Wroclaw University of Science and Technology, Faculty of Technical and Engineering, Poland \\ ${ }^{3}$ Center of Modern Technologies Ltd, Wroclaw, Poland
}

\begin{abstract}
The issue of measuring and determining the calorific value of fuels is related to thermodynamic analysis of the effects of the combustion process aimed at determining the amount of heat transferred to the environment. Currently, there are several methods for determining the calorific value of fuels and their methodology is related to the type of fuel being analyzed. These methods are quite complicated and require the use of specialized measuring equipment. The energy demand of modern civilization along with the need to protect the natural environment prompts the search for new ways to generate energy directed at sources other than conventional fossil fuels. Technologies related to the use of biogas, synthesis gas obtained in biomass or waste gasification processes are being introduced. The use of these fuels in industrial processes of generating heat and electricity requires caloric stability of the fuel. The caloric stability of the fuel is necessary to ensure the stability of thermal energy conversion processes that translate directly into the set values of generated electricity using electric machines. One way to assess the energy quality of a fuel is to measure its calorific value. There are several methods for determining the calorific value of a fuel, but they all require special measurement conditions. The article presents the author's concept of a calorimeter dedicated to the analysis of the calorific value of gaseous fuels.
\end{abstract}

\section{Introduction}

The energy demand of modern civilization along with the need to protect the natural environment prompts the search for new ways to generate energy directed at sources other than conventional fossil fuels. Technologies related to the use of biogas, synthesis gas obtained in biomass or waste gasification processes are being introduced. The use of these fuels in industrial processes of generating heat and electricity requires caloric stability of the fuel. The caloric stability of the fuel is necessary to ensure the stability of thermal energy conversion processes that translate directly into the set values of generated electricity using electric machines [1-8]. One way to assess the energy quality of a fuel is to

*Corresponding author: jaroslaw.markowski@put.poznan.p1 
measure its calorific value. There are several methods for determining the calorific value of fuel.

The calorific value can be determined on the basis of knowledge of the chemical composition of the fuel. This method is based on the assumption that the calorific value of the fuel is equal to the sum of the product of the individual components in the compound calorific value and their percentage content in the fuel. This method allows obtaining an approximate value, because it was assumed that the elements appear as single ones and does not take into account the fact that they occur in the fuel in the form of chemical compounds that have their heat of creation affecting the value of the calorific value. These methods obtain the highest accuracy of calorific value determination for hard coal. For gaseous fuels, the calorific value $\left(W_{o p}\right)$ can be determined from approximate formulas $\left[\mathrm{MJ} / \mathrm{m}^{3}\right]$.

$$
W_{o p}=\sum_{i=1}^{n}\left[\left(W_{o p}\right)_{i} \cdot \mu_{i}\right]
$$

where:

$\left(W_{o p}\right)_{i}$ - calorific value of the i-th gas component,

$\mu_{i} \quad-$ volume share of the $\mathrm{i}$-th gas component.

The calorific value of gas can be determined using a Junkers calorimeter. It is a flow device, mainly used to determine the heat of combustion and the calorific value of gaseous fuels. Combustion takes place at a constant pressure equal to the atmospheric pressure. The measurement is carried out under steady-state conditions of the combustion gas flow, with a constant cooling water flow rate. Under steady-state conditions, the calorimeter's internal energy and temperatures at individual points on the calorimeter are constant. The method consists in determining the heat released during the combustion of a certain amount of gaseous fuel in the calorimeter. The heat released is picked up by the water flowing through the calorimeter. The set consists of two main units: a laboratory gas meter with a water cooler and a gas pressure regulator. The requirements for the measurement are also room requirements. It should be bright, airy and heated, with a capacity of at least $20 \mathrm{~m}^{3}$. The room temperature should be in the range of $15-30^{\circ} \mathrm{C}$, and during the measure-ment should be set with an acceptable range of variation of $\pm 1^{\circ} \mathrm{C}$. Water feeding the calorimeter should be taken from the water supply network or from the water tank located above the calorimeter.

Examples of guidelines regarding the implementation of calorific value measurement with the use of a Junkers calorimeter emphasize the complicated methodology of the study. The indicated measurement guidelines cannot be met in the case of measurements carried out in industrial conditions outside the laboratory. Therefore, the concept of an industrial calorimeter has been developed that can meet the conditions for obtaining a satisfactory measurement result with small requirements for the conditions of measurement.

\section{The concept of measuring the calorific value of gas}

The idea of measuring calorific value in a Junkers calorimeter is to determine the value of heat received from the fuel burned in the environment of the gases, from which it is transferred as an intermediate element to the liquid flowing through the calorimeter housing. The key in this measurement is to obtain measurement stability understood as stabilizing the temperature of the cooling medium flowing with a constant mass flow value. In this method, the cooling factor is water, which has an air density 1000 times higher and about 4 times higher specific heat. In the proposed concept it is intended to omit the coolant in the calorimeter, which in the case of the Junkers Calorimeter is water. However, it is 
proposed to measure the value of heat supplied to the air supplied to the space of the fuel combustion chamber while ensuring a stoichiometric process of gas fuel combustion. The general operating principle of the device is shown in the diagram (Fig. 1).

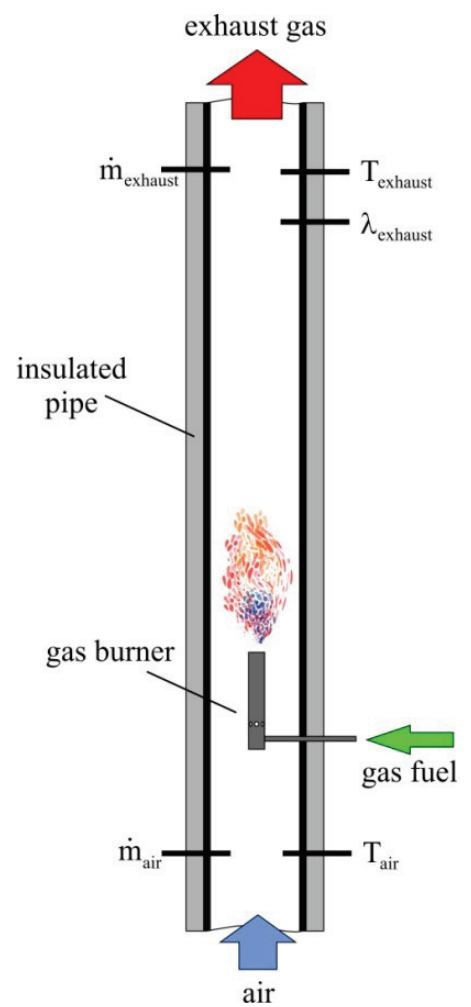

Fig. 1. Diagram of the gas calorimeter concept.

The flow-through combustion chamber is a thermally insulated circular pipe. A burner was placed inside, supplied from the outside with analyzed gas fuel and supplied with air flowing inside the pipe. The amount of air flowing inside the burner and the space of the flame-covered pipe should be adequate to ensure a stoichiometric process of fuel combustion. The assessment of the calorific value of gas in the proposed concept consists in the analysis of the amount of heat supplied to the air flowing in a thermally insulated pipe. The value of heat $Q$ supplied to air can be determined using the following equations:

$$
Q=m_{\text {air }} \cdot c_{p} \cdot \Delta T
$$

and

$$
Q=m_{f u e l} \cdot W_{o p} \cdot \zeta
$$

where:

$$
\begin{array}{ll}
Q & - \text { heat }[\mathrm{J}], \\
m_{\text {air }} & - \text { air mass }[\mathrm{kg}], \\
m_{\text {fuel }} & - \text { fuel mass }[\mathrm{kg}], \\
c_{p} & - \text { specific heat of air at constant pressure }[\mathrm{J} /(\mathrm{kg} \cdot \mathrm{K})], \\
\Delta T & - \text { temperaturę change }[\mathrm{K}], \\
W_{o p} & - \text { calorific value }[\mathrm{J} / \mathrm{kg}],
\end{array}
$$




\section{$\zeta \quad$ - efficiency of the combustion process [-].}

By comparing equations 2 and 3 with each other, we obtain the relationship described by the equation:

$$
m_{\text {air }} \cdot c_{p} \cdot \Delta T=m_{\text {fuel }} \cdot W_{\text {op }} \cdot \zeta
$$

this equation can be transformed in such a way as to obtain relationship 5 that allows determining the calorific value of the fuel:

$$
W_{o p}=\frac{m_{\text {air }} \cdot c_{p} \cdot \Delta T}{m_{\text {fuel }} \cdot \zeta} .
$$

To assess the calorific value of gaseous fuel in accordance with the proposed calorimeter concept, it is necessary to determine the values of other quantities included in the presented relationships. It is necessary to determine the mass of the medium flowing through the combustion chamber. It should be taken into account that the flowing medium is initially air, then it participates in the oxidation of the supplied fuel in the stoichiometric process and eventually takes the form of exhaust gases. Assuming that there is no loss of medium mass in the measuring system, this situation can be written by the general equation:

$$
m_{\text {air }}+m_{\text {fuel }}=m_{\text {exhaust }}
$$

Due to the fact that we are dealing with a continuous flow of factor, equation 6 should be written as follows:

$$
\dot{m}_{\text {air }}+\dot{m}_{\text {fuel }}=\dot{m}_{\text {exhaust }}
$$

To determine the air mass flow $\left(m_{\text {air }}\right)$, its flow measurement must be used, similarly to the mass flow stream $\left(m_{\text {exhaust }}\right)$. During these measurements, it is necessary to take into account the value of the air inlet temperature to the device and the temperature of the exhaust gas in the outlet channel of the device. Knowing these values and taking into account equation 7 , one can determine the mass flow of fuel fed to the measuring system $\left(m_{\text {fuel }}\right)$.

When measuring oxygen concentration in flue gas with the use of e.g. an oxygen sensor, one should strive to ensure a stoichiometric combustion process in which the excess air ratio $\lambda=1$, and for which the efficiency of the combustion process $\zeta=1$. During the combustion process of fuels, the mass of air always has a significantly greater proportion of fuel. Therefore, often for simplicity, the process analysis is carried out taking into account the specific heat $\mathrm{cp}$ of air.

By determining the values of individual parameters by measuring in a stabilized, stoichiometric process of combustion of gaseous fuels and using the presented relationships, it is possible to determine the calorific value of the supplied gaseous fuel.

\section{Summary}

The issue of measuring and determining the calorific value of fuels in currently used measurement methods requires the use of very expensive and demanding testing equipment, and the required conditions for the measurement are large in relation to ensuring an appropriate laboratory environment and qualified research staff. This situation is quite complicated in relation to the reality of the widespread demand for ongoing assessment of calorific value in industrial installations such as various types of biogas 
plants and energy installations converting biogas into heat or electricity. Responding to existing measurement problems in this area, the concept of a measuring device was proposed, which can contribute to obtaining the expected results of calorific value measurement in a much less complicated way. The concept presented in the article is the starting point for further considerations and laboratory work, the results of which will be presented in subsequent articles.

\section{References}

1. H. Yang, Q. Fu, Xu. Cheng, R. K. K. Yuen, H. Zhanga, Investigation of the flammability of different cables using pyrolysis combustion flow calorimeter, ELSEVIER Procedia Engineering 62, 778-785, (2013)

2. K. R. Overdeep, T. P. Weihs, Design and functionality of a high-sensitivity bomb calorimeterspecialized for reactive metallic foils, Journal of Thermal Analysis and Calorimetry 122(2):787-794. DOI: 10.1007/s10973-015-4805-8.

3. EL. Dreizin, Metal-based reactive nanomaterials, Prog EnergyCombust Sci. 35, 141-167, (2009)

4. AS.Rogachev, Exothermic reaction waves in multilayer nano-films, Russ Chem Rev. 77, 21-37, (2008)

5. M. Ribeiro da Silva, G. Pilcher, L. Santos, L. Lima, Calibration and test of an aneroid mini-bomb combustioncalorimeter, J. Chem. Thermodyn. 39, 689-697, (2007)

6. J. Mentado, E. Mendoza, Calibration and testing of an isoperibolicmicro-combustion calorimeter developed to measure the enthalpyof combustion of organic compounds containing C, H, $O$ and N, J. Chem. Thermodyn. 59, 209-213, (2013)

7. A. Rojas-Aguilar, An isoperibol micro-bomb combustion calorimeter for measure-ment of the enthalpy of combustion. Application tothe study of fullerene C60. J. Chem. Thermodyn. 34, 1729-1743, (2002)

8. M. Sakiyama, T. Kiyobayashi, Micro-bomb combustion calorimeter equipped with an electric heater for aiding completecombustion, J. Chem. Thermodyn. 32, 269-279, (2000) 